\author{
Graziano BASSI*
}

\title{
Descrizione di una nuova Catoptria Hübner, 1825 dell'Italia centrale, con note sul suo gruppo-specie di appartenenza (Lepidoptera: Pyraloidea, Crambidae, Crambinae)
}

\begin{abstract}
Riassunto: Viene descritta Catoptria apenninica n. sp. dell'Appennino Abruzzese (Italia centrale), appartenente al gruppo di permutatella. Si definisce la storia e la consistenza del gruppo e viene proposto di ristabilire lo status di bona species per le seguenti entità: Catoptria casalei Bassi, 1999 sp. rev., Catoptria gozmanyi Błeszyński, 1956 sp. rev., Catoptria olympica Ganev, 1983 sp. rev.

Abstract: Description of a new Catoptria Hübner, 1825 from Central Italy, with notes on its species-group (Lepidoptera: Crambidae, Crambinae). Catoptria apenninica $\mathrm{n}$. sp. of the permutatella group is described from the Apennines of the Abruzzi (Central Italy). The history and size of the group are revisited and on suggest to re-establish as bona species the following taxa: Catoptria casalei Bassi, 1999 sp. rev., Catoptria gozmanyi Błeszyński, 1956 sp. rev., Catoptria olympica Ganev, 1983 sp. rev.
\end{abstract}

Key words: Crambini; Italy; new species; permutatella species-group; the Abruzzi.

\section{INTRODUZIONE}

Nonostante la lepidotterofauna europea e italiana sia considerata abbastanza ben conosciuta, a seguito di ricerche mirate vengono ancora alla luce specie interessanti o addirittura inedite. In questo caso si descrive una nuova Catoptria Hübner, 1825 appartenente al gruppo permutatella Błeszyński catturata in quota nell'Appennino abruzzese durante una spedizione di ricerca effettuata congiuntamente ai dottori Giorgio Baldizzone e Renato Barbero di Asti.

\section{MATERIALI E METODI}

Nello studio del materiale la tecnica di dissezione adottata è quella descritta da Robinson (1976), mentre la terminologia segue Klots (1970) e Kristensen (2003). Gli adulti sono stati fotografati con una Nikon D300, mentre i preparati microscopici sono fotografati con una Canon S120. L'holotypus, catturato a vista in pieno giorno nella prateria alpina, vicino ad alcune rocce, è conservato nella collezione dell' Autore (CGB).

\section{Catoptria apenninica n. sp.}

Holotypus 9 : Abruzzo, Serra Rocca Chiarano (Aquila), 2250 m, 18.VII.1990, leg. G. Bassi, preparato microscopico 2675 Bassi, CGB.

DERIVATIO NOMINIS: Il nome deriva dal Latino apenni- $n u s-i=$ Appennini, e si riferisce all'area geografica in cui è stato catturato l'esemplare.

Diagnosi: La specie più vicina a $C$. apenninica $\mathrm{n}$. sp. (Fig. 1) è C. europaeica Bleszynski (Fig. 2), ma l'adulto se ne differenzia per le minori dimensioni (26,7 $\mathrm{mm}$ contro i $30 \mathrm{~mm}$ medi della femmina di $C$. europaeica); 1'ala anteriore ha colore più chiaro, stria mediale lievemente più ampia, area costale subterminale gialla più grande e non divisa in due; l'ala posteriore è bruna con frange bianche, mentre in $C$. europaeica è grigio-bruna con frange avorio. Nei genitali femminili (Figg. 3 e 4) la lamella antevaginalis è più piccola e meno sporgente, l'ostium bursae e la prima parte del ductus bursae sono decisamente più ampi e lo sternite dell'VIII segmento addominale meno sporgente rispetto a C. europaeica (Fig. 5).

Descrizione: Femmina (Fig. 1): Apertura alare 26,7 $\mathrm{mm}$. Antenne filiformi, brune con costa dorata. Palpi labiali lunghi 3 volte il diametro dell'occhio composto, bianchi ai lati e internamente, bruno ocra al centro. Palpi mascellari subtriangolari, bruno giallastri con punta bianca. Fronte bianca, tondeggiante, chiaramente sporgente oltre l'occhio. Torace bianco bordato di bruno. Patagia brune. Ali anteriori bruno gialle; costa bruno gialla con ampia area subapicale più chiara; stria mediale bianca divisa da bande brune e

*Graziano Bassi, Via Sant’Agostino 51, 10051, Avigliana (TO), Italia. E-mail: graziano.bassi@alice.it 
parzialmente circoscritta da linee brune; parte distale della vena $\mathrm{Cu} 2$ segnata da una linea di scaglie bianche; margine dorsale bianco nella metà prossimale; termen segnato da linea bruno nerastra; frange dorate con base bianca. Ali posteriori castano con breve linea bianca mediale lungo la vena $\mathrm{m} 2$; frange bianco puro. Addome bruno giallastro. Zampe anteriori brune; zampe mediali e posteriori crema.

Genitali femminili (Figg. 3 e 4): Papillae anales subtriangolari. VIII segmento addominale ampio, sclerificato, con sclerite dotato di due processi lamellari simmetrici che risultano al di sotto dello sterigma, laterali all'asse del ductus bursae. Ostium bursae ampio, a forma di coppa. Sterigma con lamella antevaginalis tronca, sporgente ventralmente, Ductus bursae lungo

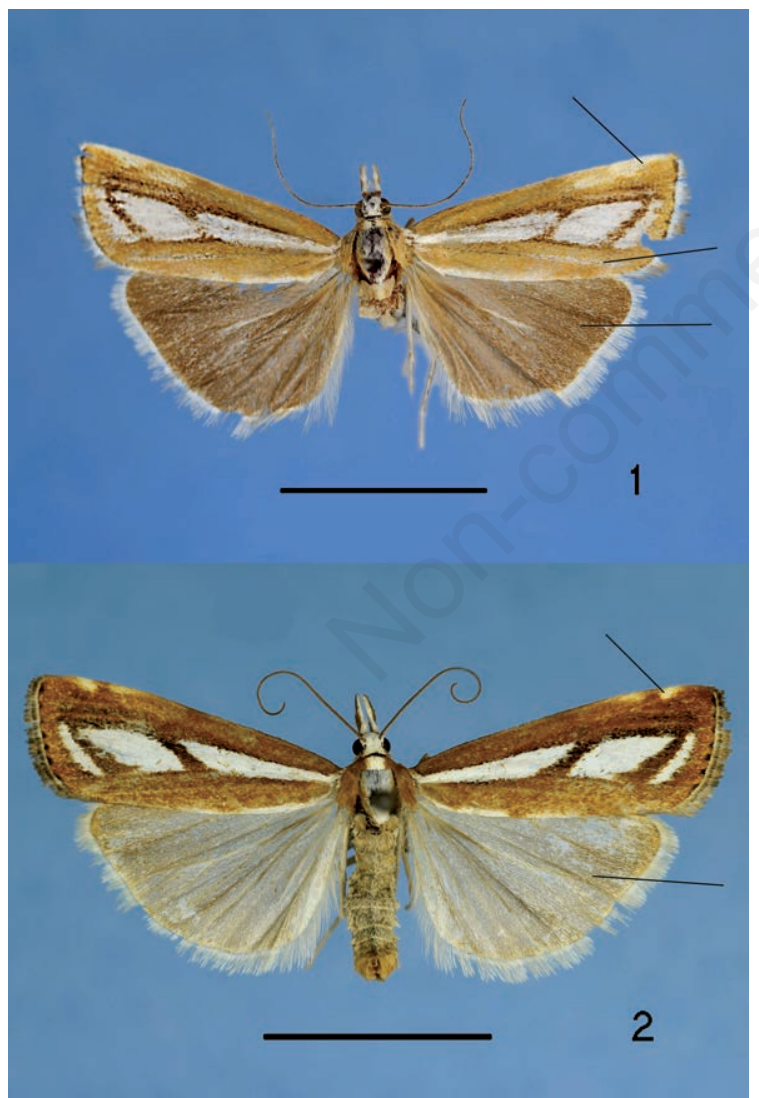

Figg. 1-2. Catoptria sp., adulti. 1 - Catoptria apenninica $\mathrm{n}$. sp., Holotypus. 2 - Catoptria europaeica Błeszyński, femmina, Italia, Piemonte, Valsusa, Venaus (TO), $1860 \mathrm{~m}$, 26.VII.2013, leg. Bassi. Scala $=10 \mathrm{~mm}$. Le linee indicano i principali caratteri differenziali evidenziati nel testo. due volte il corpus bursae, stretto, sclerificato e corrugato fino a $2 / 3$ della sua lunghezza. Corpus bursae subovale, con un unico signum arrotondato e cosparso di microplacche. Il ductus seminalis nasce dal ductus bursae, alla fine della sclerificazione.

Maschio sconosciuto.

DifFusione: Nota solo della località tipica in Abruzzo. Note: $C$. apenninica n. sp. appartiene al gruppo permutatella sensu Błeszyński (1957: 323), che conta 26 specie note (su 84 Catoptria conosciute), di cui 9 ( $C$. europaeica Błeszyński, C. luctiferella (Hübner), $C$. myella (Hübner), C. orobiella Huemer \& Tarmann, $C$. osthelderi (de Lattin), C. pyramidella (Treitschke), $C$. spatulella (Turati), C. spatulelloides Błeszyński, $C$. speculalis Hübner sono sicuramente presenti in Italia

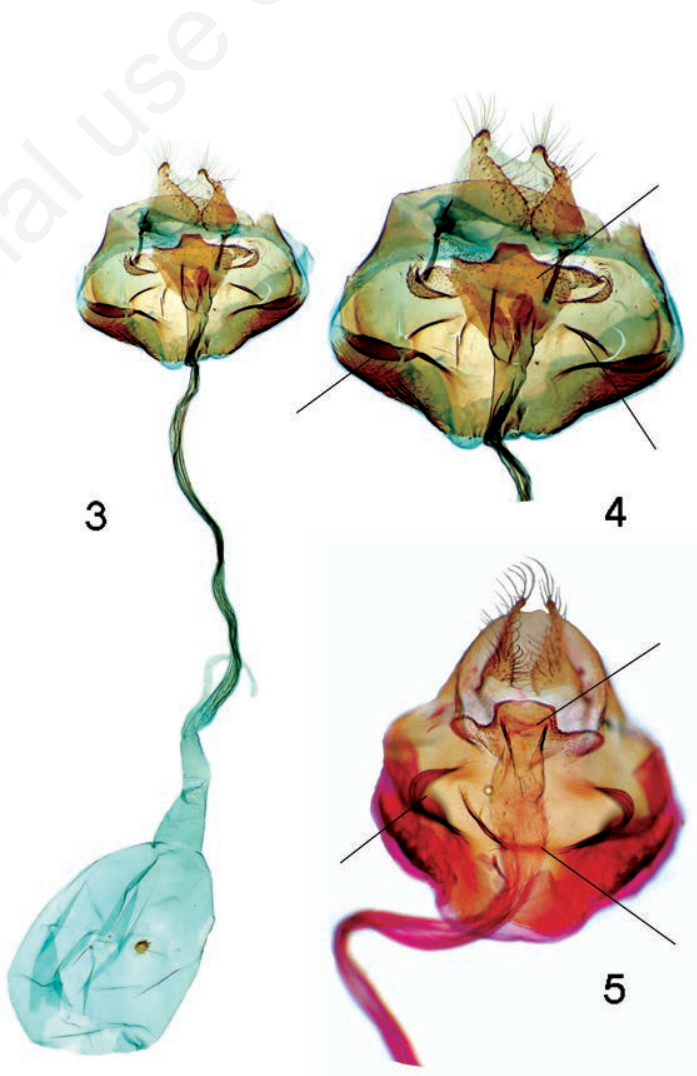

Figg. 3-5. Catoptria sp., apparati genitali femminili. 3 - $\mathrm{Ca}$ toptria apenninica n. sp., Holotypus, intero apparato. 4 - $\mathrm{Ca}$ toptria apenninica $\mathrm{n}$. sp., Holotypus, sterigma, ostium bursae e sclerificazioni dell'VIII segmento addominale. 5 - Catoptria europaeica Bleszynski, Piemonte, Colle di Sampeyre (CN), 1.VIII.1986, leg. Bassi \& Scaramozzino, sterigma, ostium bursae e sclerificazioni dell'VIII segmento addominale. Le linee indicano i principali caratteri differenziali evidenziati nel testo. 
e un'altra, $C$. permutatella Hübner, è stata recentemente segnalata per il territorio italiano (Pinzari et al., 2010) (materiale non esaminato). In Italia il gruppo è tipicamente legato alle aree montane e collinari e in genere dimostra una spiccata tendenza all'endemizzazione, dimostrata dalle 4 specie, compresa apenninica, descritte dal 1965 ad oggi. Anche al di fuori del territorio nazionale vi è la tendenza alla speciazione del gruppo considerato, già oggetto di pubblicazione specifica (Bassi, 1999) e ancora in fase di approfondimento, anche con tecniche su base molecolare.

Il gruppo permutatella è stato ed è oggetto di una certa confusione, forse per la forte somiglianza nell'habitus e le poche differenze negli apparati genitali. Błeszyński lo istituì nel 1957, basandosi sull'esame degli apparati genitali e distinguendolo al suo interno in tre sottogruppi a seconda della presenza di stria longitudinale dell'ala anteriore spezzata (I, permutatella sensu strictu), o unica (II, pyramidella), e colore di fondo bianco (III, acutangulella). Peraltro lo stesso autore (1965: 262 e segg.) dette al gruppo di specie il nome "myella-gruppe", senza più distinguere i sottogruppi, coerentemente con l'omogeneità della struttura dei genitali nella varie specie. Ad aumentare la confusione Leraut $(2012: 542,547)$ ha sinonimizzato, senza dare spiegazioni frutto di studio approfondito, ben 3 specie. In questo lavoro si usa il nome più vec- chio attribuito al gruppo specie (permutatella) e propongo il reintegro allo status di bona species per le seguenti entità: Catoptria casalei Bassi, 1999 sp. rev., Catoptria gozmanyi Błeszyński, 1956 sp. rev., Catoptria olympica Ganev, 1983 sp. rev. Tali specie sono state studiate in modo esteso e puntuale (Bassi, 1999: 351, 352, Figg. 1, 3, 4, 11, 12, 29-35 e 37) e le caratteristiche che distinguono le une dalle altre sono costanti, nell'habitus ma soprattutto negli apparati genitali. Nel genitale maschile la lunghezza e la forma dei processi presenti nella valva sono fondamentali per la determinazione, mentre nei genitali femminili le differenze sono concentrate a livello di forma e dimensione dell'VIII segmento addominale, sterigma, ostium e ductus bursae. È inoltre curioso che Leraut stesso (2012: 541) cita A. (sic!) gozmanyi come buona specie comparandola con C. permutatella, pur sinonimizzandola con C. myella nella pagina successiva.

\section{RINGRAZIAMENTI}

Ringrazio i compagni di viaggio Giorgio Baldizzone e Renato Barbero e tutti coloro, la cui lista sarebbe troppo lunga, che hanno permesso, con consigli, prestiti e doni di materiale, la crescita della mia conoscenza sulle Crambinae. Un ringraziamento anche ai due referees per i costruttivi suggerimenti.

\section{BIBLIOGRAFIA}

BASsI G., 1999 - Note sulle Crambinae Paleartiche con la descrizione di tre nuove specie (Lepidoptera: Pyraloidea, Crambinae). Shilap. Revista de Lepidopterologia, 27: 349-360.

BŁESZYŃSKI S., 1957 - Studies on the Crambidae (Lepidoptera). Part XIV. Revision of the European species of the generic group Crambus F. s. 1. Acta Zoologica Cracoviensia, 1: 161-622.

BŁesZyŃSKi S., 1965 - Crambinae. pp. i-1, 1-553, pls. 1-133. In: AmSel H.G., Gregor F, ReISSER H, (eds). Microlepidoptera Palaearctica 1 (1-2). Georg Fromme \& Co., Wien.

KLOTS A.B., 1970 - Lepidoptera. In: TUXEN SL (Ed.). Taxonomist's glossary of genitalia in insects. (Second revised and enlarged edition). Munksgaard, Copenhagen, pp 115-130.

KRISTENSEN N.P., 2003 - Skeleton and muscles: adults. In: Lepidoptera, moths and butterflies. Vol. 2. Morphology, physiology, and development. Handbook of Zoology IV (36). Walter de Gruyter, Berlin, New York, pp 39-131.

Leraut PJA, 2012 - Zygènes, Pyrales 1 et Brachodides. Papillons de nuit d'Europe, Verrières-le-Buisson 3: 599 pp.

PinZari M., PInZARI M., ZilLi A., 2010 - Deep Lepidopterological exploration of Mt. Cagno and surroundings (Central Italy), a restricted mountain massif And hotspot for butterfly and moths diversity (Lepidoptera). Bollettino dell'Associazione Romana di Entomologia, 65: 3-383.

RoBinson G.S., 1976 - The preparation of slides of lepidoptera genitalia with special reference to the Microlepidoptera. Entomologist's Gazette, 27: 127-132. 